\title{
Closure to "Effect of Multilevel Inverter Supply on Core Losses in Magnetic Materials and Electrical Machines"
}

\author{
Paavo Rasilo, Aboubakr Salem, Ahmed Abdallh, Frederik De Belie, Luc Dupré, \\ and Jan. A. Melkebeek, Senior Member, IEEE
}

$\mathrm{T}_{\mathrm{t}, \mathrm{s}}$ HE AUTHORS appreciate Prof. Ruderman's additions to the literature review of the paper. Indeed, [1] and [2] provide a theoretical approximation for the core losses of magnetic components and electrical machines subject to multilevel pulse-width modulated (PWM) voltage supply. The main emphasis is on the classical eddy-current losses, which are assumed to be the dominating PWM-induced additional loss component. In [1], the additional eddy-current losses are derived to be proportional to the mean-squared supply voltage ripple. In [2], the normalized mean-squared (NMS) voltage ripples are derived for multilevel converters with an arbitrary number of voltage levels.

The assumption that the eddy-current loss is the dominating additional iron loss component is supported by our numerical simulations for the ring core (Table II of the paper) and for the synchronous machines (Figs. 8 and 11). It is noteworthy that in the larger machine, the additional damper-winding loss is several times larger than the additional iron loss. In [2] it is predicted that increasing the number of voltage levels reduces the core losses, which is also our conclusion. However, Table II and Figs. 8 and 11 show significant dependency of the eddy-current loss on the switching frequency. This contradicts with the suggestions of [1] and [2], which base on the fact that the NMS voltage ripple is independent from the switching frequency at least on practical frequency ranges. The proportionality of the eddy- current loss to the NMS voltage is only valid under the assumption that the winding resistance and leakage inductance as well as the skin effect of the eddy currents in the core lamination are negligible, and thus the problem in practice is not so trivial, as also mentioned in [2]. Our numerical iron-loss calculations model the skin effect, and the windings are accounted for by supplying the model with the measured flux-density waveforms (Table II) or by coupling the finite element model to the circuit equations (Figs. 8 and 11). When the skin effect becomes non-negligible, magnetic nonlinearity and the interdependency of the eddy currents on the hysteresis and excess losses [3] make analytical prediction of the losses difficult if not impossible. Nevertheless, [1] and [2] offer interesting reference formulas for further iron-loss measurements and simulations in devices under PWM supply.

\section{REFERENCES}

[1] A. Ruderman and R. Welch, "Electrical Machines PWM loss evaluation basics," in Proc. Int. Conf. on Energy Efficiency in Motor Driven Systems, (EEMODS), Vol. 1, pp. 58-68, Sep. 2005.

[2] A. Ruderman and B. Reznikov, S. Busquets-Monge, "Asymptotic time domain evaluation of a multilevel multiphase PWM converter voltage quality," IEEE Trans. Ind. Electron., Vol. 60, No. 5, pp. 1999-2009, May 2013.

[3] E. Dlala, A. Belahcen, J. Pippuri, A. Arkkio, "Interdependence of Hysteresis and Eddy-Current Losses in Laminated Magnetic Cores of Electrical Machines," IEEE Trans. Magn., Vol. 46, No. 2, pp. 306-309, Feb. 2010.

\footnotetext{
Manuscript received October X, 2015.

P. Rasilo is with the Department of Electrical Engineering, Tampere University of Technology, P.O. Box 692, FI-33101 Tampere, Finland and also with the Department of Electrical Engineering and Automation, Aalto University School of Electrical Engineering, FI-00076 Espoo, Finland (e-mail: paavo.rasilo@tut.fi).

A. Abdallh, A. Salem, F. De Belie, L. Dupré, and J. A. Melkebeek are with the Department of Electrical Energy, Systems and Automation, Ghen University, Technologiepark 913, BE-9052 Gent-Zwijnaarde, (e-mail: $\quad$ ahmed.abdallh@ieee.org; $\quad$ SalemMohamed.AboubakrSalem @UGent.be; $\quad$ Frederik.DeBelie@UGent.be; luc.dupre@ugent.be; jan.melkebeek@ugent.be).

A. Abdallh is also with the Electrical Power and Machines Department, Cairo University, EG-12613 Giza, Egypt.

Digital Object Identifier ....
} 